\title{
ANALISIS GEOMÁTICO DE BLOQUES ARADORES Y LÓBULOS EN LOS PUERTOS DE ALIVA (PICOS DE EUROPA, CORDILLERA CANTÁBRICA)
}

\author{
José Juan de SANJOSÉ BLASCO (jiblasco@unex.es) \\ Departamento de Expresión Gráfica, Universidad de Extremadura \\ Enrique SERRANO CAÑADAS (serranoe@fyl.uva.es) \\ PANGEA, Departamento de Geografía, Universidad de Valladolid \\ Manuel GÓMEZ LENDE, (manuelglende@hotmail.com) \\ PANGEA, Departamento de Geografía, Universidad de Valladolid.
}

Recibido: 04/01/2016

Aceptado: 13/03/2016

RESUMEN: En Áliva (Picos de Europa, Montaña Cantábrica) se ha realizado un control de la dinámica de lóbulos de solifluxión, y bloques aradores. Los Puertos de Áliva se sitúan entre el grupo Peña Vieja, en el macizo central, y el macizo oriental o de Andara. Constituido por una sucesión de pizarras negras con intercalaciones de conglomerados calcáreos, calizas y areniscas, la Formación Lebeña forma una depresión donde domina el modelado glaciar. En las laderas destacan los lóbulos, las terracillas, bloques aradores, acarcavamientos, deslizamientos de ladera y desprendimientos. Con el objetivo de conocer la dinámica geomorfológica actual en los Puertos de Áliva, y establecer la importancia de los procesos asociados al hielo en la transferencia de sedimentos y la erosión de suelos, se ha realizado un control geomático de lóbulos, bloques aradores, cárcavas y deslizamientos. Estos se complementan con registros térmicos de suelos, análisis de la Estación Meteorológica Automática y cartografía geomorfológica. Las observaciones geomáticas se han realizado entre 2008 y 2015. La técnica empleada ha sido GPS-RTK y TLS. La zona se ha dividido en tres áreas de trabajo: parcela 1, medición de tres lóbulos; parcela 2, observación de dos bloques aradores y un lóbulo y parcela 3 , con medición de cuatro bloques aradores. El desplazamiento de los lóbulos y bloques aradores, con promedios menores de $0,5 \mathrm{~cm} \mathrm{a}^{-1}$ denotan una baja actividad. El régimen térmico del suelo señala la inexistencia o moderada presencia del hielo en el suelo, por ello se plantea la hipótesis de que correspondan a geoformas heredadas del pasado reciente, con condiciones ambientales más favorables a la actividad periglaciar y con una dinámica actual asociada a la acción nival.

PALABRAS CLAVE: Geomática, laderas, periglaciar, Cordillera Cantábrica.

GEOMATIC SURVEY OF PLOUGHING BLOCKS AND SOLIFLUCTION LOBES IN THE ALIVA MEADOWS (PICOS DE EUROPA, CANTABRIAN MOUNTAIN)

ABSTRACT: Dynamic control of solifluction lobes and ploughing blocks was carried out in the Áliva area (Picos de Europa, Cantabrian Mountains). Áliva meadows are three little valleys between the Central massif and the Ándara massif drained by the Duje river. The depression is 
formed by conglomerate, sandstone and slates of the Lebeña Formation, deeply shaped by glaciers. On the slopes lobes, terracettes, ploughing blocks, badlands and slides are commons. A geomatic survey was carried out to monitor geomorphic processes, and establish the importance of processes linked to the frozen ground in sediment transfer on slopes. The study was complemented with the installation of several loggers in order tomonitor the ground thermal regime and the analysis of EMAs. The research was conducted between 2008 and2015usingtwo techniques: GPS-RTK and TLS. Three study sites including solifluction lobes, ploughing blocks and badlands were analyzed. The lobes and ploughing blocks showed displacement rates lower than $0.5 \mathrm{~cm} \mathrm{a}^{-1}$. Thesemovement rates suggest a moderate activity of mass wasting rates in an area where frozen ground conditions are almost absent. We hypothesize that these landforms are inheritedfrom recent past conditions when the environmental conditions were most favorable to periglacial activity. Nowadays, geomorphic activity is linked to nival processes.

KEY WORDS: Geomatic survey, slopes, periglacial, Cantabrian mountains.

\section{INTRODUCCIÓN}

En los Picos de Europa la nieve y el hielo estacional en el suelo y el subsuelo son los elementos más representativos de la criosfera, seguidos por los heleros y las cuevas heladas. Los heleros y el hielo estacional se sitúan por encima de los 1.900 m s.n.m., y sólo la nieve se extiende a cotas bajas, con importancia socioeconómica y como riesgo natural. La nieve es un elemento clave en la alta montaña y componente transversal de la criosfera, que influye en las aguas superficiales y el comportamiento térmico del suelo, los flujos biogeoquímicos y la dinámica de los ecosistemas (De WALle y RANGO, 2008; ADAMS y HAMLET, 2009; MARSHALL, 2011). Las características del manto nival determinan, en parte, los procesos periglaciares y desempeñan un papel central en las temperaturas del suelo y el régimen anual de la escorrentía (GARCíA RUIZ et al., 2011; LÓPEZ-MORENO et al., 2008, 2009), de modo que detectar la presencia de hielo en el suelo y los suelos helados estacionales es esencial para comprender los procesos geomorfológicos en la montaña supraforestal. En ambientes periglaciares de alta montaña, la temperatura del suelo determina buena parte de los procesos geomorfológicos y su comportamiento a lo largo del año establece los umbrales críticos que los desencadenan.

Para conocer la dinámica geomorfológica actual y establecer la importancia de los procesos asociados al hielo es necesario establecer su actividad o inactividad y la existencia o inexistencia de hielo en el suelo, de modo que nos indiquen si son procesos activos, y su efectividad en la transferencia de sedimentos. Para ello, las técnicas geomáticas ofrecen unas precisas posibilidades para el control de diferentes ambientes fríos entre los que se encuentran los procesos periglaciares de laderas (SANJOSÉ et al., 2014).

El estudio del comportamiento térmico de los suelos permite establecer la presencia o ausencia de hielo en el suelo, la magnitud y duración de la pene- 
tración de la onda de frío o de las temperaturas por debajo de $0{ }^{\circ} \mathrm{C}$, y en definitiva, detectar la eficacia de los procesos actuales asociados al hielo en el suelo, o su posible inactividad o herencia de periodos pasados, y su utilidad como geoindicadores de cambio en la alta montaña supraforestal. La hipótesis de trabajo parte de que la actividad de estas formas puede estar asociada a cambios climáticos recientes, con intervención del hielo o la nieve en su funcionamiento, para lo cual debemos establecer su génesis y los procesos que intervienen; o como respuestas a la intervención y usos humanos, también recientes.

El objetivo de este trabajo es conocer la dinámica geomorfológica actual en los Puertos de Áliva, mediante la aplicación de técnicas geomáticas, y establecer la importancia de los procesos asociados al hielo en la transferencia de sedimentos y la erosión de suelos.

\section{LA ZONA DE ESTUDIO}

El sector denominado como Puertos de Áliva constituye un área relativamente deprimida entre el Grupo Peña Vieja, del macizo central, y el macizo oriental o de Andara, en los Picos de Europa (FIGURA 1). Los Puertos de Áliva se extienden entre 1.450 y $1.850 \mathrm{~m}$ de altitud y están constituidos por una sucesión de pizarras negras con intercalaciones de conglomerados calcáreos, calizas y areniscas, la Formación Lebeña, que contrasta con el entorno calcáreo de las Formaciones Picos de Europa y Calizas de Montaña (MARQuínEZ, 1989, 1992). Estas últimas generan paredes verticales en los dos macizos que circunvalan los Puertos de Áliva, y también las cumbres del Cueto de Juan Toribio, klippe tectónica, que divide Áliva y Las Salgardas.

El modelado dominante es glaciar, con presencia de circos glaciares, umbrales sobre calizas y un importante sistema morrénico frontal, en Las Salgardas, y lateral de obturación en el Duje (GONZÁLEZ-TRUEBA, 2007a; SERRANO et al., 2012, 2013) que modelan el valle.

La litología ha determinado la presencia de una zona deprimida, con laderas de pendiente más moderada, la existencia de fuentes en los contactos con las calizas que generan una escorrentía superficial e incisiones fluviales. Las laderas poseen un pequeño recubrimiento coluvionar en el que se desarrollan formas netamente diferenciadas del entorno kárstico. Entre estas formas sobresalen los lóbulos, las terracillas, bloques aradores, cárcavas, deslizamientos de ladera y desprendimientos.La nitidez de las formas de erosión y de solifluxión indican una actividad actual o muy reciente que ha sido señalada con anterioridad en el diferentes lugares, y particularmente en Áliva, donde se han 
establecido posibles grados de actividad y los factores geoecológicos que intervienen (MIOTKE, 1968; BROSCHE, 1978, 1994; SERRANO y GONZÁLEZ TruebA, 2004; GonZÁleZ TruebA, 2007). Los Puertos de Áliva forman amplias praderías ocupadas por ganado durante gran parte del verano, así como una intensa actividad turística (vehículos $4 \times 4$, excursionistas, bicicletas de montaña, Refugio-Hotel de Áliva, Chalet Real).

Figura 1. Localización de Áliva

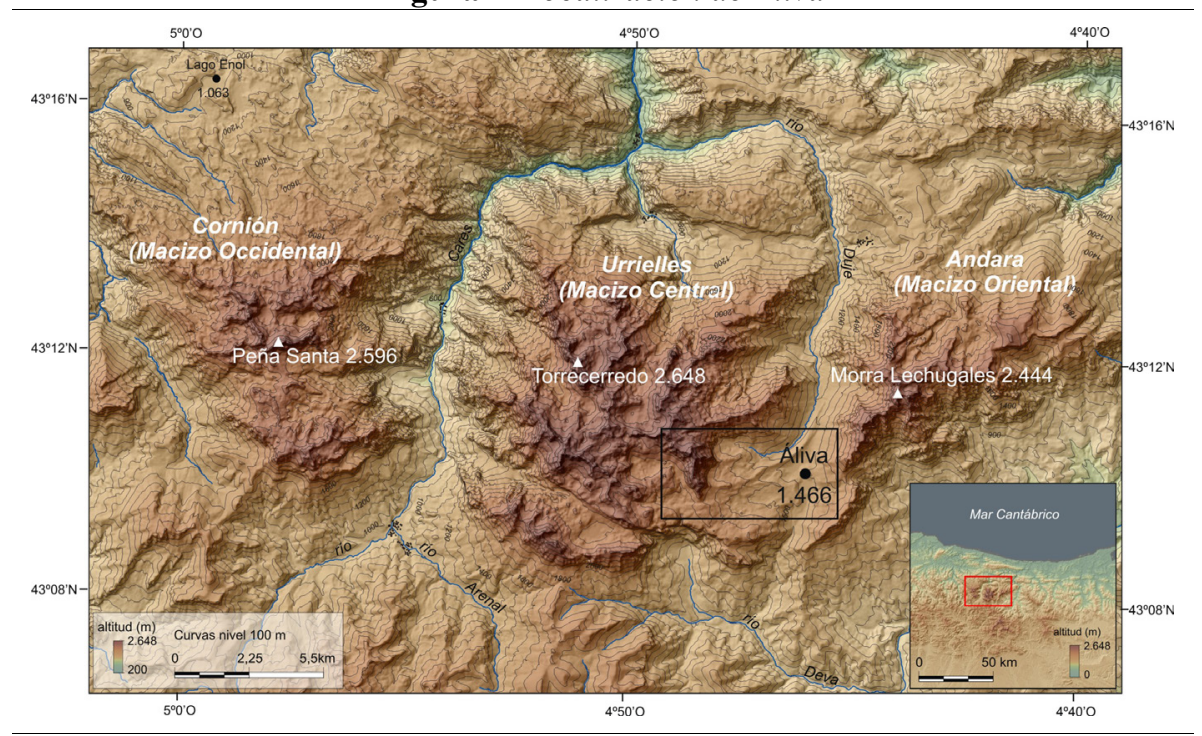

FUENTE: Elaboración de los autores

\section{MATERIAL Y MÉTODOS}

Para el estudio de los procesos activos y su control temporal se han aplicado diferentes técnicas cartográficas, geomáticas y de control térmico orientadas a conocer sus caracteres dinámicos, ambientales y las relaciones entre ellos, que se explican a continuación.

\section{1. Control térmico:}

Se han analizado las estaciones meteorológicas más próximas (GÓMEZ LENDE, 2015) y se han instalado termómetros de suelos (PISABARRO et al., 2015). En la estación meteorológica automática de El Cable, se han estimado las temperaturas medias anuales, el número de días con temperaturas por debajo de $0^{\circ} \mathrm{C}$, el índice de helada y los ciclos de hielo/deshielo (TABLA 1). Para el control térmico de suelos se han utilizado termómetros a 1.115, $1.720 \mathrm{y}$ $1.865 \mathrm{~m}$ de altitud, en Áliva y Lloroza, áreas muy próximas (TABLA 2). Se 
han utilizado microsensores térmicos iButton y UTL-Geotest AG (Universal TemperatureLogger) data-logger con precisión centesimal, y se han establecido fases térmicas conforme a la metodología de Delaloye (2004) y calculado el índice de helada (Ih) y ciclos de hielo-deshielo (PISABARRO et al., 2015).

Tabla 1. Principales parámetros climáticos en el entorno de Áliva

\begin{tabular}{ccccccc}
\hline Estación & Tipo & Altitud $(\mathrm{m})$ & TMAA & Ih & \multicolumn{2}{c}{ Ciclos $H / d H$} \\
\hline El Cable & $\mathrm{A}$ & 1823 & $6^{\circ} \mathrm{C}$ & 178 & $20-30$ & Oct/Abr \\
Lloroza & $\mathrm{S}$ & 1865 & $6,3^{\circ} \mathrm{C}$ & 55 & 8 & Mar/Dic \\
\hline
\end{tabular}

Tabla 2. Localización y parámetros de los termómetros de suelos

\begin{tabular}{lccccc}
\hline Lugar & Altitud & Días $T^{a}$ & Días $T^{a}<$ & Ih & Días con hielo \\
& $(\mathrm{m})$ & $<0^{\circ} \mathrm{C}$ & $-2^{\circ} \mathrm{C}$ & & deshielo \\
Lloroza & 1865 & 79 & 2 & 55,17 & 8 \\
\hline Áliva & 1720 & 34 & 0 & 2,64 & 6 \\
\hline Fuentedé & 1115 & 0 & 0 & 0 & 0 \\
\hline
\end{tabular}

\section{III.2. Control geomático:}

En Áliva se han realizado observaciones geomáticas empleando GPS-RTK en 4 lóbulos de solifluxión, 6 bloques aradores, 1 cárcava y 1 deslizamiento, con una periodicidad anual sin interrupciones, desde agosto de 2008 hasta agosto de 2015. Para el desarrollo de la técnica de GPS-RTK se posiciona una base fija y estable, en la cual se estaciona el GPS de referencia. A partir de esta base fija se toman datos con el GPS móvil sobre cada uno de los puntos de interés (lóbulos, cárcavas y bloques aradores). El error posicional en la medida de cada punto está entre 1 y $2 \mathrm{~cm}$, por ello si el valor de desplazamiento de los elementos analizados es inferior a $2 \mathrm{~cm}$, se considera que no existe movimiento. La zona se ha dividido en tres áreas de trabajo: parcela 1, medición de tres lóbulos; parcela 2, observación de dos bloques aradores y un lóbulo y parcela 3, con medición de cuatro bloques aradores (FIGURA 2). En cada lóbulo se realiza un perfil longitudinal y se mide el frente (inflexión inferior), y en los bloques aradores se mide el contorno del bloque y el frente del lóbulo generado por el bloque.

Para el control de los procesos geomorfológicos se han empleado un láser escáner terrestre «ScanStation C10» (Leica) en el deslizamiento del Chalet Real con una densidad de malla de escaneado de $5 \times 5 \mathrm{~cm}$ para una distancia de 100 metros. Se utiliza este TLS de medio alcance que mide distancias en un rango de 1,5 a $300 \mathrm{~m}$, con una precisión nominal de $+/-6 \mathrm{~mm}$ a una distancia de $50 \mathrm{~m}$ con iluminación normal y en condiciones de reflectividad. El campo de visión vertical tiene una amplitud de $270^{\circ}$ sexagesimales y $360^{\circ}$ en el plano 
horizontal. El software utilizado para la grabación, alineación de las nubes de puntos y tratamiento de los datos es LeicaCyclone 7.3®.

Figura 2. Localización de las tres áreas de trabajo en Áliva:1) medición de tres lóbulos; 2) dos bloques aradores y un lóbulo; 3) cuatro bloques aradores; 4) deslizamiento del Chalet Real.

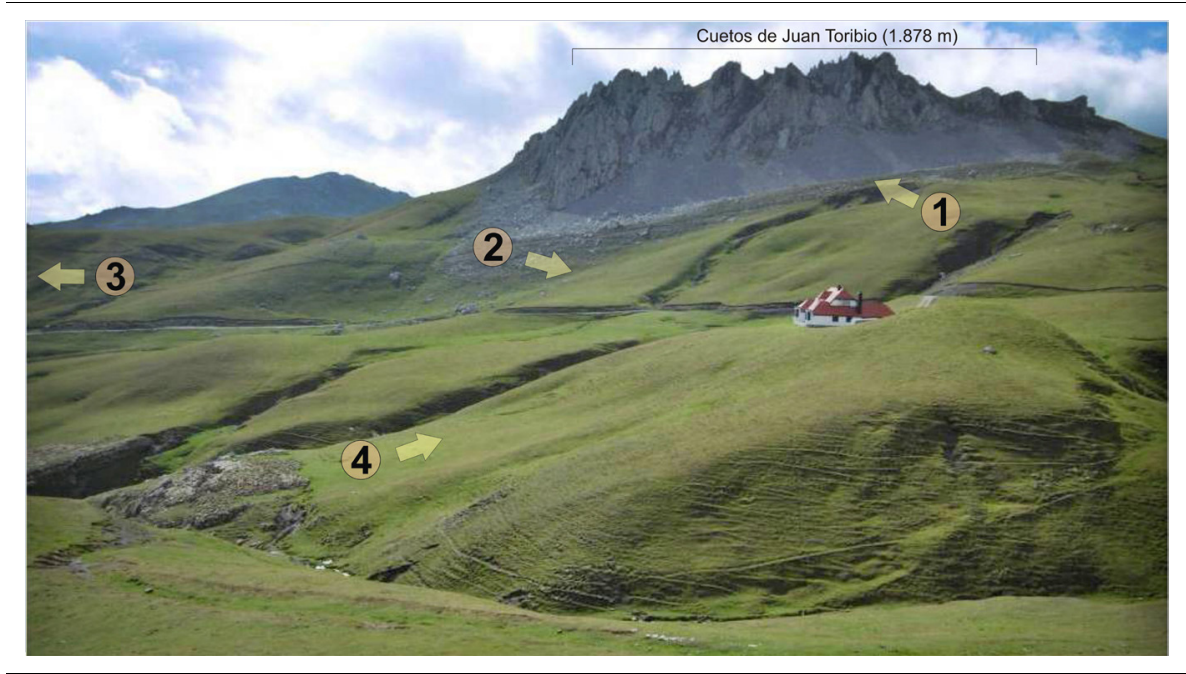

FUENTE: Elaboración de los autores

\section{3. Mapa geomorfológico:}

A partir de los mapas existentes a E.1/25.000 (SERRANO Y GONZÁLEZTRUEBA, 2004; GONZÁLEZ-TRUEBA, 2007a) se han elaborado mapas geomorfológicos detallados (E. 1/10.000) del entorno de Áliva para inventariar y referenciar las formas de modelado y los procesos de ladera y establecer relaciones espaciales y altitudinales.

\section{RESULTADOS}

Las laderas de Áliva muestran procesos de erosión concentrada y movimientos en masa que remodelan la morfología glaciar modelada durante las crisis frías pleistocenas (FIGURA 3). Durante la última fase glaciar, constatada por las morrenas de Cueto de Juan Toribio y de Áliva, estos pequeños glaciares aportarían materiales proglaciares de tipo fluvio torrencial que rellenaron las cubetas. En algunos casos los deslizamientos de ladera obturarían los arroyos y cubetas, generando un periodo de relleno con depósitos lacustres de poco espesor. 
Finalmente, todas las formaciones o el sustrato presentan depósitos de arcillas, gravas y cantos aristados con lentejones heterométricos que denotan actividad de laderas, con aportes al fondo de valle y regularización de las mismas. Con posterioridad se desarrollan los suelos sobre esta formación.

Figura 3. Vista de Áliva desde los Cuetos de Juan Toribio. En primer término acarcavamientos, al fondo el arroyo Resalao y el valle del Duje.

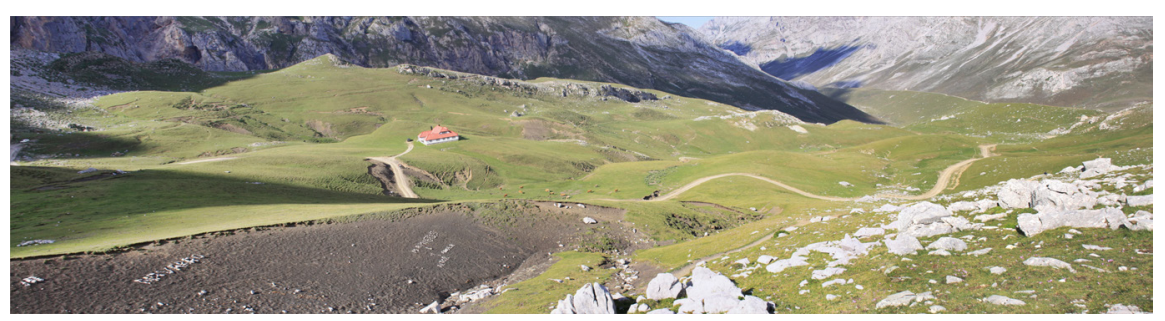

FUENTE: Elaboración de los autores

Figura 4. Formas y procesos geomorfológicos en el sector superior de Áliva. 1, morrena. 2, taludes y conos de derrubios. 3, laderas regularizadas. 4, incisiones lineales. 5, acarcavamientos. 6, lóbulos de solifluxión. 7, deslizamientos de ladera. 8, bloques dispersos. 9, Chalet Real.

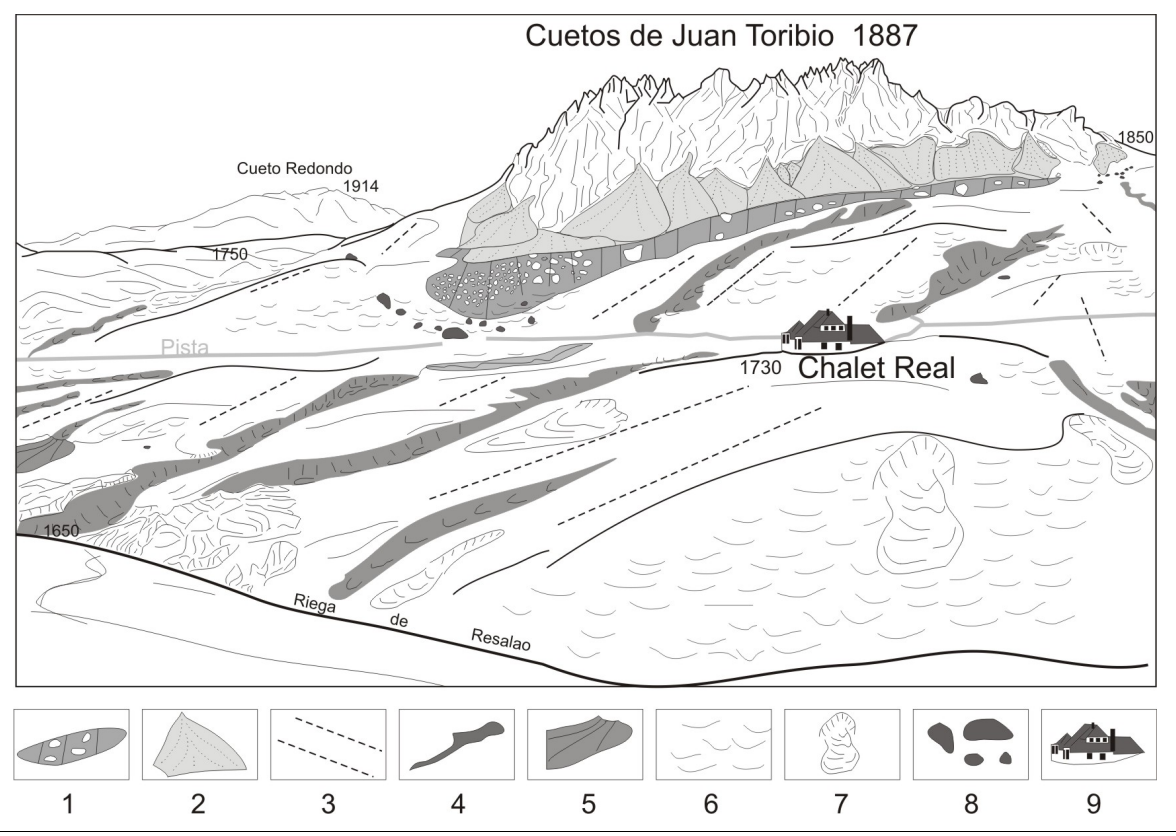

FUENTE: Elaboración de los autores 
Los canales de incisión, de carácter remontante, que culminan con acarcavamientos aún parcialmente activos señalan una nueva fase de desequilibrio, si bien en muchos casos están en parte rellenas de depósitos solifluidales que denotan una escasa dinámica torrencial o fluvial, y donde se alojan los lóbulos más activos.

Las laderas regularizadas, las incisiones y las morrenas están afectadas por procesos de solifluxión recientes, bloques aradores, lóbulos de solifluxión, terracillas y solifluxión laminar, que han sido objeto de mediciones puntales. Las últimas fases de inestabilidad se deben asociar al Holoceno, a falta de una mayor precisión temporal (FIGURA 4).

Figura 5. Ejemplos de las geoformas monitorizadas en Áliva. A) Lóbulo de solifluxión en el área 2. B) Deslizamiento del Chalet Real. C) Bloques aradores en el área 3. D) Lóbulo de solifluxión en el área 1.

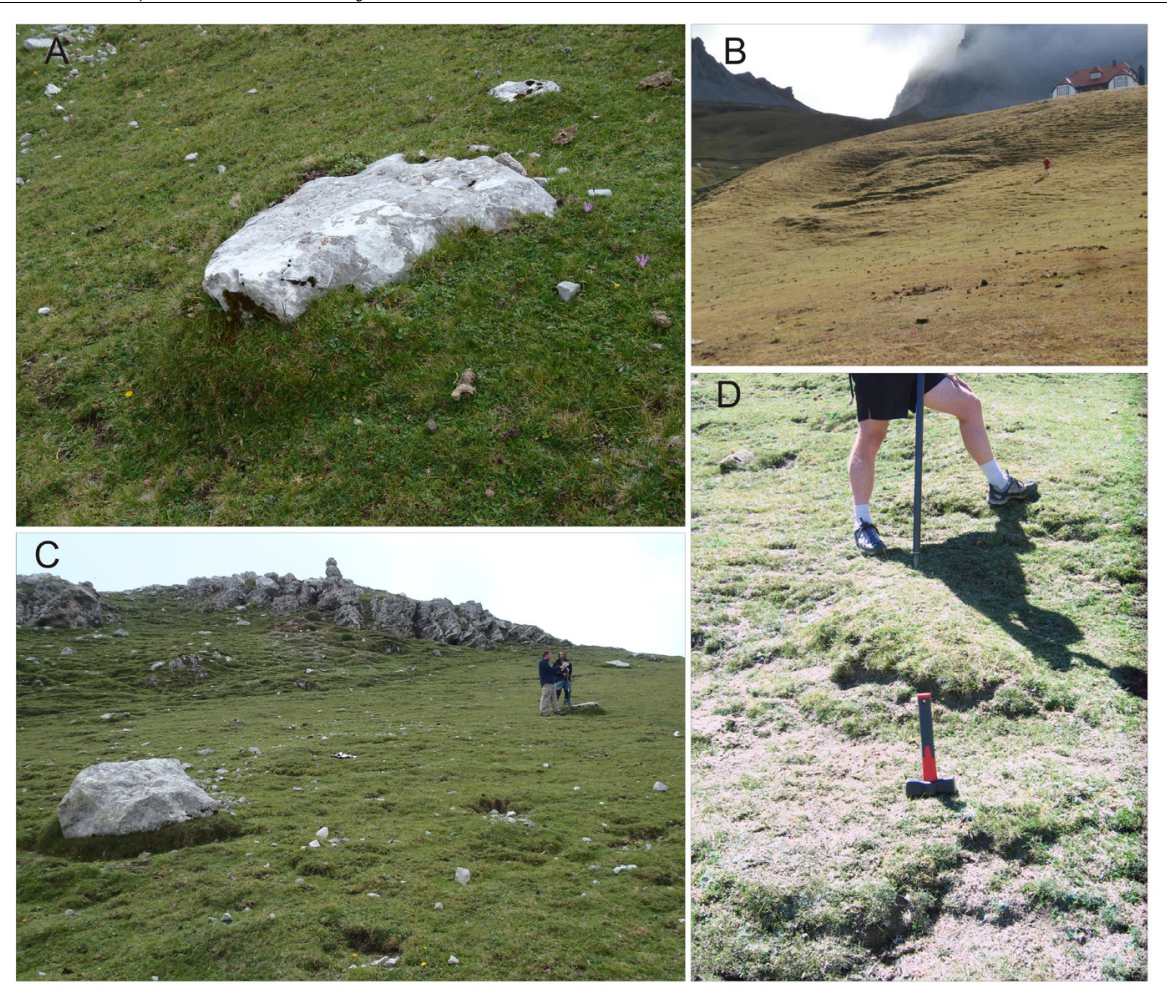

FUENTE: Elaboración de los autores

Los lóbulos y bloques aradores (FIGURA 5) han registrado para un periodo de siete años (2008-2014) un desplazamiento inferior a $4 \mathrm{~cm}$, con un promedio 
anual inferior a $0,5 \mathrm{~cm} \mathrm{a}^{-1}$. No puede afirmarse que su dinámica sea inexistente, ya que hay una tendencia en dirección a la pendiente, pero es muy moderada, y en muchos casos en el margen de error de los aparatos de medida. El gráfico muestra la deformación y muy reducido avance de las formas estudiadas, principalmente las asociadas a los cauces, donde la disponibilidad hídrica y la presencia de finos favorecen su desplazamiento (FIGURAS 6 y 7). Los lóbulos muestran procesos de adelgazamiento y deterioro con cambios milimétricos. Sin embargo, los bloques aradores muestran movimientos similares, pero con desplazamientos netos que señalan una dinámica existente, pero muy atenuada.

Figura 6. Detalle del desplazamiento medido mediante GPS-RTK en lóbulos en Áliva

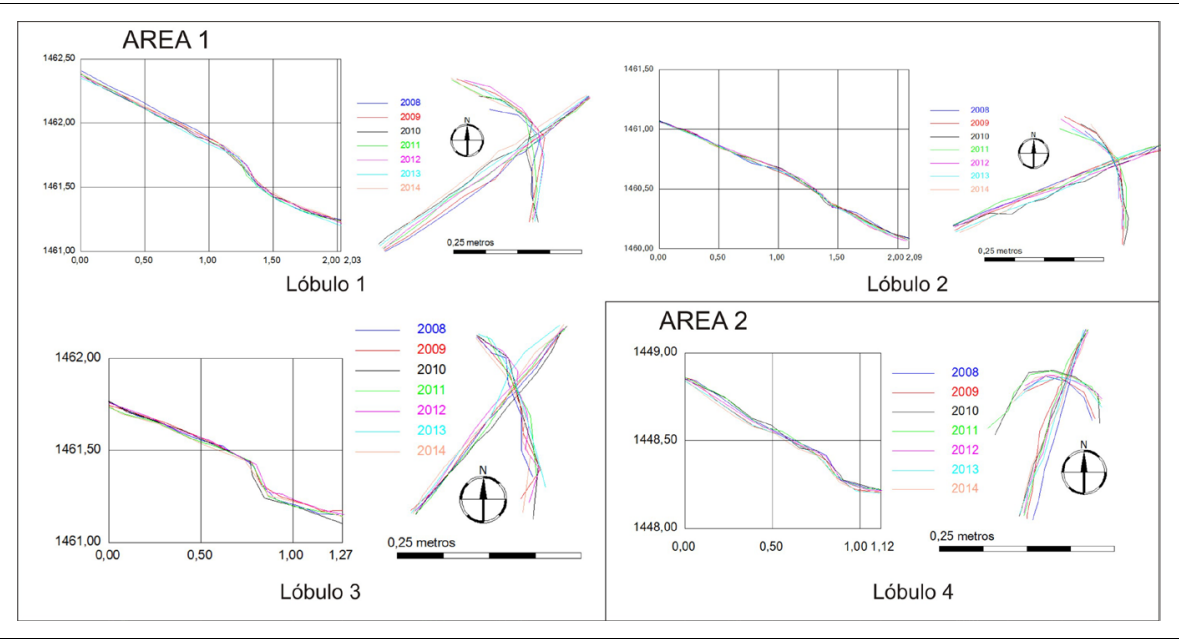

FUENTE: Elaboración de los autores

En Áliva y Lloroza el índice de helada en el suelo (TABLA 1) es muy bajo (Ih: 2,64, en Áliva) y en Fuente Dé, a $1.115 \mathrm{~m}$, es nulo (PISABARRO et al., 2015), lo que señala un periodo muy breve de congelamiento. Este hecho está conforme con las temperaturas medias anuales del aire, pues ningún mes presenta temperaturas medias anuales del aire por debajo de $0^{\circ} \mathrm{C}$. El manto nival es muy variable, entre 2 y 7 meses por año a una cota de 1.900 m (TABLA 3 ), lo que se concreta en exposiciones del suelo a las temperaturas atmosféricas de invierno muy variable. Los años con un manto nival muy breve, la superficie del suelo permanece expuesta hasta tres meses de invierno.

La estación de El Cable muestra entre 20 y 50 ciclos de hielo-deshielo anuales, todos ellos concentrados en el periodo otoñal durante 5-6 días antes del recubrimiento nival. En el suelo los ciclos de hielo-deshielo son inferiores a 
10 (H/dH: 6 ciclos, en Áliva, TABLA 2) y se producen entre enero y abril, asociados a la inestabilidad del manto nival y las temperaturas de invierno. Las temperaturas del suelo en invierno se sitúan por encima de $0{ }^{\circ} \mathrm{C}$, bajo el efecto Zero curtain, y sólo a final de invierno y primavera hay descensos bruscos y breves que indican la influencia atmosférica bajo un delgado manto nival.

Figura 7. Desplazamientos de bloques aradores mediante GPS-RTK en Áliva

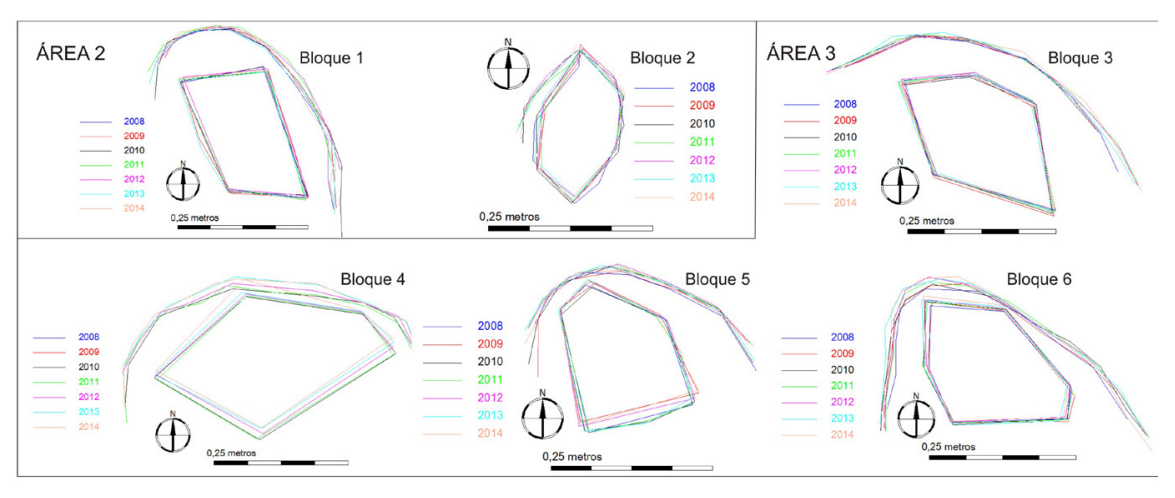

FUENTE: Elaboración de los autores

Tabla 3. Duración del manto nival en La Vueltona-Lloroza (1850-1950 $\mathrm{m}$ s $\mathrm{n}$ m)

\begin{tabular}{ccc}
\hline Año & $N^{o}$ Meses & Periodo \\
\hline 2010 & 5 & Diciembre a Mayo \\
2011 & 4 & Enero a Mayo \\
2012 & 2 & Marzo a mayo \\
2013 & 7 & Diciembre a Junio \\
2014 & 6 & Enero a junio \\
\hline
\end{tabular}

FUENTE: Elaboración de los autores

Tabla 4. Presencia de hielo en el suelo por debajo de los $1900 \mathrm{~m}$

\begin{tabular}{ccccl}
\hline Altitud $(m)$ & $\begin{array}{c}\text { TMAA } \\
\left({ }^{\circ} \mathrm{C}\right)\end{array}$ & $\begin{array}{c}\text { Meses con } \\
T^{a} \leq 0^{\circ} \mathrm{C}\end{array}$ & Manto nival & \multicolumn{1}{c}{ Suelos helados } \\
\hline $1900-1850$ & 6,3 & 3 & Inestable & $\begin{array}{l}\text { Límite altitudinal suelos } \\
\text { helados estacionales }\end{array}$ \\
$1800-1700$ & 7 & 0 & Inestable & Improbables \\
$1200-1100$ & 11,7 & 0 & Inestable & No existen \\
\hline
\end{tabular}

FUENTE: PISABARRO et al., 2015

En Áliva, se puede establecer que no todos los años existen suelos helados estacionales (ninguno durante la fase medida), y que el hielo está presente sólo ocasionalmente en ciclos muy cortos, asociado al manto nival inestable y dis- 
continuo que perdura cinco meses al año. La presencia suelos helados estacionales y hielo en el suelo en Picos de Europa, se localiza por encima de los 1.850-1.900 m de altitud, lo que sitúa Áliva por debajo de este umbral (TABLA 4). Denota, pues, una dinámica asociada en mayor medida a la nieve y los procesos de fusión que a la presencia de hielo en el suelo.

Figura 8. Régimen térmico del suelo en Áliva (2005-2007) a 1.720 m de altitud

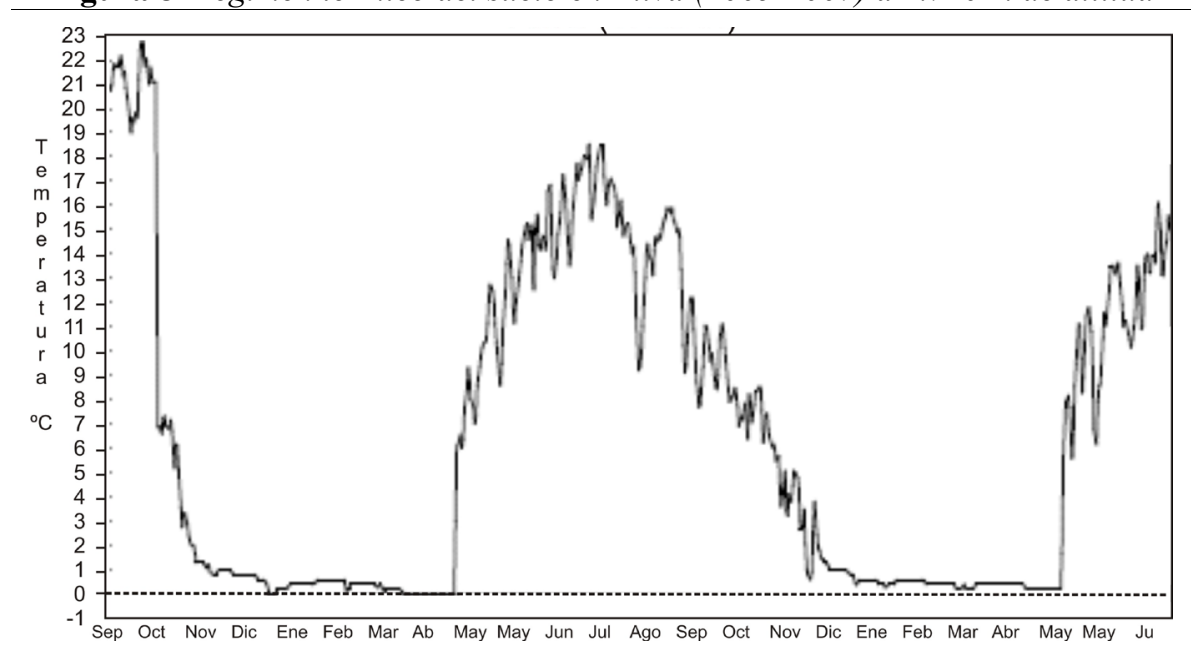

FUENTE: Elaboración de los autores

\section{DISCUSIÓN}

Los escasos ciclos de hielo y deshielo en el suelo (TABLA 2), debido a la nieve que reduce e incluso anula estos procesos, implica que por debajo de los $1.700 \mathrm{~m}$, el hielo es un elemento escaso y la crioclastia y la crioturbación muy poco eficaces. Todo está acorde con el escaso dinamismo de las formas, lóbulos, bloques aradores y cárcava, que cuando son más activos, fundamentalmente procesos erosivos, están asociados a la actividad humana (pistas, minería y sendas de excursionistas).

Sin embargo, la buena conservación de las formas, unida a la inexistencia de helada en el suelo en la actualidad, permiten establecer la hipótesis de que los procesos dominantes en Áliva son los nivales y la solifluxión, asociada a la fusión, las fuentes y a las elevadas precipitaciones de primavera, verano y otoño. Las formas y depósitos pueden estar en relación con herencias del pasado reciente, cuando estos procesos fueran más eficaces. Los bloques aradores se definen como formas asociadas a la presencia de hielo y de procesos gelifluidales (FRENCH, 2007) propios de ambientes periglaciares, si bien en 
las montañas españolas estos se asocian a procesos nivales sin presencia de hielo.

Por otra parte, los lóbulos están condicionados por los factores climáticos, hidrológicos, geológicos y morfotopográficos (MATSUOKA, 2001), y en la alta montaña peninsular se ha constatado (BROSCHE, 1984; OLIVA y GÓMEZ ORTIZ, 2011; OLIVA et al., 2014) su dependencia de la pendiente, la cobertura vegetal, la disponibilidad hídrica, la duración y espesor del manto nival y el régimen térmico del suelo, pero en ambos casos asociados a suelos helados estacionales. El aporte hídrico procedente de la fusión nival satura o sobresatura los depósitos de finos, que pierden cohesión y reptan, en todo caso asociado a la presencia de suelo helado estacional en profundidad (HARRIS et al., 1997; MATSUOKA, 2001; OliVA et al., 2008, 2009), de modo que el mayor desplazamiento se produce durante el deshielo, con procesos sincrónicos de crioreptación y gelifluxión. En Picos de Europa, en las zonas altas sobre derrubios calcáreos, los desplazamientos solifluidales varían entre 0,21 y 1,88 $\mathrm{cm} \mathrm{a}^{-1}$ (BROSCHE, 1994), y en ambientes periglaciares marítimos de Escocia

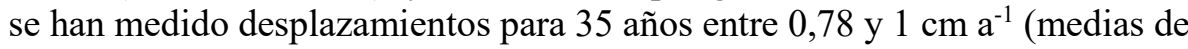
$0,88 \mathrm{~cm} \mathrm{a}^{-1}$ ), que sugieren similares velocidades superficiales de los lóbulos de solifluxión y los bloques aradores, y ambas menores que en las zonas desprovistas de vegetación, donde la reptación por agujas de hielo (pipkrake) es el principal componente de la solifluxión (BALLANTYNE, 2013). En Sierra Nevada, en ambientes con suelos helados estacionales, los movimientos de los lóbulos son de $\sim 0,5 \mathrm{~cm} \mathrm{a}^{-1} \mathrm{y}$ su desplazamiento se corresponde con procesos de centenares a miles de años, y dinámicas actuales asociadas a la saturación por fusión nival (OLIVA et al., 2008, 2009, 2014).

En Áliva, el dinamismo de los lóbulos, bloques aradores y cárcavas, induce a pensar que las formas y los procesos son herencias de condiciones pasadas, aunque recientes, que propiciaron la presencia de suelos estacionales, hoy con una dinámica muy atenuada a favor de la fusión nival, en particular a neveros tardíos, y la disponibilidad hídrica en las canales, relacionadas con la fusión y las fuentes, pero en ningún caso a la presencia de hielo estacional. Las condiciones para su génesis pueden remontarse, bien a la Pequeña Edad del Hielo, bien al Holoceno, sin que en el momento actual se pueda establecer más precisión. La presencia humana desde la antigüedad, con fases de pastoreo en pastos naturales de altura desde hace 6.500 años (DíEz CASTILLO, 1997, 2011) y la existencia de una fuerte presión ganadera con una deforestación generalizada en la Edad Media (AZCUÉNAGA, 1978), podría implicar la desestabilización reciente de las laderas de Áliva. El descenso térmico de $\sim{ }^{\circ} \mathrm{C}$ en Picos durante la Pequeña Edad del Hielo (GonZÁLEZ TRUEBA, 2006) y la conserva- 
ción de las formas, sugieren asociarlo a este periodo, con una fase de desarrollo de 400-500 años, si bien sólo se puede establecer como hipótesis, pues para ciclos de miles de años, se podría remontar al Holoceno.

\section{CONCLUSIONES}

En Áliva, se ha constatado la inexistencia de suelos helados estacionales y la escasez de hielo en el suelo por los reducidos ciclos de hielo deshielo y el bajo índice de helada.

El control dinámico de los bloques aradores no muestra tendencias significativas y no permite afirmar que son activos actualmente. En uno de ellos se ha apreciado una ligera basculación pero acorde con la acumulación de finos diferencial en una determinada porción del bloque. Los lóbulos muestran un promedio anual $<5 \mathrm{~mm} \mathrm{a}^{-1}$. Todos los lóbulos existentes están condicionados por el contenido de humedad, la textura de la formación superficial y la superación del límite plástico, procesos propios de dinámicas nivales. Sólo en uno de ellos se produce una tendencia al adelgazamiento de su porción superior (lóbulo 2 de la parcela 1), consecuencia de procesos erosivos más que de desplazamientos.

Las formas solifluidales, bloques aradores y lóbulos, se pueden relacionar con procesos periglaciares del pasado, como formas heredadas de periodos más activos del pasado reciente, hoy retocadas por procesos principalmente nivales. La combinación de ambos procesos puede ser la responsable de la dinámica y morfología de laderas, si bien son necesarios estudios y dataciones más detalladas, en marcha en la actualidad, para aclarar su génesis y edad. De este modo, las formas y procesos actuales están asociados a periodos de mayor efectividad del pasado, bien por hielo en el suelo, bien por la acción humana que desestabiliza las laderas.

En la actualidad en los Puertos de Áliva los procesos asociados al hielo en el suelo son excepcionales, las huellas periglaciares no son plenamente activas y su morfodinámica se fundamenta en procesos nivales y la solifluxión. La fusión nival satura las formaciones superficiales desencadenando su dinámica.

\section{AGRADECIMIENTOS}

Este trabajo es una contribución al proyecto CGL2015-68144-R, financiado por el Ministerio de Economía y Competitividad. Deseamos agradecer a Fernando Berenguer, María González, Juan José González Trueba y Manuel Sánchez su ayuda en los trabajos de campo. 


\section{BIBLIOGRAFÍA}

ADAMS, J. C. Y HAMLET, A. F. (2009): «Implications of global climate change for snowmelt hydrology in the twenty-first century». HydrologicalProcesses, 23(7), 962-972.

AzCuÉNAGA, J. (1978): «Las ordenanzas del Puerto de Áliva». Anales del Instituto de Estudios Agropecuarios, 3, 157-214.

BALLANTYNE, C.K. (2013): «A 35-year record of solifluction in a maritime periglacial environment». Permafrost and Periglacial Processes, 24 (1), 56-66.

BROSCHE, K.U. (1978): Beiträgezumrezenten und vorzeitlichenperiglazialen Formenschatz auf der Iberischen Halbinsel. Abhandlungen des Geographischen Instituts, Sonderhefte, Band I. Geographischen Instituts der Freien Universität Berlin. Berlin, $285 \mathrm{pp}$.

BRosCHE, K.U. (1994): «Ergebnisse von Abtragungsmessungen an periglazialenSolifluktionsschuttdecken in vierHochgebirgen der IbersichenHalbinsel (Picos de Europa, Peña Prieta, Sierra de Urbión und Sierra Nevada)». Eiszeittalter u. Gegenwart, 44, 28-55.

De Walle, R. Y RangO, A. (2008): Principles of snow hydrology. Cambridge University Press, Cambridge.

DELAlOYE, R. (2004): Contribution à l'étude du pergélisol de montagne en zone marginale. PhD thesis, Fac. Sciences, Univ. Fribourg, GeoFocus, 10.

Diez CastiLlo, A. (1997): «Utilización de los recursos en la marina y Montañas cantábricas: una prehistoria ecológica de los valles de Deva y Nansa». Illunzar, 3, 1190.

Diez CAStiLlo, A. (2010): «Nuevas tecnologías y viejas piedras: un repaso al megalitismo en Cantabria». Illunzar, 7, 7-35.

FRENCH, H.M. (2007): The periglacial environment. Wiley, Chichester.

GARCÍA RUIZ J.M.; LÓPEZ MORENO, J.I.; SERRANO, S.M.; BEGUERÍA, S. Y LASANTA, T. (2011): «Mediterranean water resources in a global change scenario». Earth Science Reviews, 105 (3-4), 121-139.

Gómez Lende, M. (2015): Cuevas heladas en Picos de Europa. Climas, morfologías $y$ dinámicas. Tesis Doctoral. Universidad de Valladolid. Valladolid.

GonZÁlez TruebA, J.J.; MARTÍn MORENO, R.; MARTíneZ DE PISÓN, E.; Y SERRANO, E. (2008): «Little Ice Age glaciation and current glaciers in the Iberian Peninsula». The Holocene, 18(4), 551-568.

GONZÁLEZ TRUEBA, J.J. (2006): «Topoclimatical factors and very small glaciers in Atlantic Mountain of SW Europe: The Little Ice Age glacier advance in Picos de Europa (NW Spain)». Zeitschrift für Gletscherkunde und Glazialgeologie, 39, 115-125.

GonZÁlez TruebA, J.J. (2007a): El paisaje natural del Macizo Central de los Picos de Europa. CIMA, Consejería de Medio Ambiente, Santander.

GonZÁlez TRUeBA, J.J. (2007b): Geomorfología del Macizo Central del Parque Nacional de Picos de Europa. OAPN-Ministerio de Medio Ambiente, Madrid.

HARRIS, C.; DAVIES, M. Y COUTARD, J.P. (1997): «Rates and processes of periglacial solifluction: an experimental approach». Earth Surface Processes and Landforms, $22,849-868$. 
HARRIS, C. (2009): «Permafrost and climate in Europe: geomorphological impacts, hazard assessment and geotechnical response». Earth Science Reviews, 92(3-4), $117-171$.

López Moreno, J.I.; Goyette, S.; Beniston, M. y AlverA, B. (2008): «Sensitivity of the snow energy balance to climatic changes: implications for the evolution of snowpack in the Pyrenees in the 21st century». Climate Research, 36, 206-217.

LÓPEZ MORENO, J.I.; GOYETTE, S.; BENISTON, M. (2009): «Impact of climate change on snowpack in the Pyrenees: Horizontal spatial variability and vertical gradients». Journal of Hydrology, 374 (3-4), 384-396.

MARQUínEZ, J. (1989): «Síntesis cartográfica de la región del Cuera y los Picos de Europa». Trabajos de Geología, 18, 137-144.

MARQuínEZ, J. (1992): «Tectónica y relieve en la Cornisa Cantábrica». En The late Quaternary in the western Pyrenean region (CEARRETA, A.; UGARTE, F.M. eds.). Universidad del País Vasco, Vitoria, 143-160.

Marshall, S.J. (2011): The Cryosphere. Princeton Univ. Press, Princeton.

MATSUOKA, N. (2001): «Solifluction rates, processes and landforms: a global review». Earth Science Reviews, 55, 107-134.

MiotKe, F.D. (1968): Karstmorphologischestudien in der glacial-überformenHöhenstufe der Picos de Europa, Nordspaien. Jahrbuch der Geographischen Gesellschaft, Institut der Technischen Universitdt Hannover, Hannover, 161 pp.

OLIVA, M. Y GÓMEZ ORTIZ, A. (2011): «Factores que condicionan los procesos periglaciares de vertiente actuales en Sierra Nevada. El caso de la solifluxión». Nimbus, 27-28, 137-158.

Oliva, M.; Schulte, L. y Gómez Ortiz, A. (2008): «Solifluction lobes in Sierra Nevada (Southern Spain): morphometry and palaeoenvironmental changes». Proceedings of the IX International Conference on Permafrost. Vol. 2, IPA, University of Alaska-Fairbanks, Fairbanks, 1321-1326

Oliva, M.; Schulte, L. y Gómez OrTiz, A. (2009): «Morphometry and Late Holocene activity of solifluction landforms in the Sierra Nevada, Southern Spain». Permafrost and PeriglacialProcesses, 20 (4), 369-382.

Oliva, M., Gómez OrTiz, A., SAlvador, F., Y SAlvÁ, M. (2014). «Present-day solifluction processes in the semi-arid range of Sierra Nevada (Spain)». Arctic, Antarctic, and Alpine Research, 46 (2), 365-370.

Pisabarro, A.; Serrano, E. y GonzÁlez Trueba, J.J. (2015): «Régimen térmico de suelos del Macizo Central de Picos de Europa (España)». Pirineos. Revista de Ecología de Montaña, 170, doi: http://dx.doi.org/10.3989/Pirineos. 2015.170003.

Sanjosé, J.J.; Berenguer, F.; AtKinson, A.D.J.; De Matías, J.; Serrano, E.; GómEz OrTIZ, A.; GONZÁlez GARCíA, M. Y RiCO, I. (2014): «Geomatics techniques applied to glaciers, rock glaciers and ice-patches in Spain (1991-2012)». Geografiska Annaler, 96A, 307-321.

SERrAnO, E. y GonZÁlez TRUEBA, J.J. (2004): «Morfodinámica periglaciar en el grupo Peña Vieja (Macizo Central de los Picos de Europa, Cantabria)». Cuaternario y Geomorfología, 18 (3-4), 73-88.

Serrano, E.; GonzÁlez TruebA, J.J. y GonZÁlez GarcíA, M. (2012): «Mountain glaciation and paleoclimate reconstruction in the Picos de Europa (Iberian Peninsula, SW Europe)». Quaternary Research, 78, 303-314. 
Serrano, E.; GonzÁlez Trueba, J.J.; Pellitero, R.; GonzÁlez García, M. Y GÓMEZ LENDE, M. (2013): «Quaternary glacial evolution in the Central Cantabrian Mountains (Northern Spain)». Geomorphology, 196, 65-82. 